\title{
Cisplatin-induced hyponatremia in malignancy: comparison between brand-name and generic formulation
}

This article was published in the following Dove Press journal:

Drug Design, Development and Therapy

3 December 2014

Number of times this article has been viewed

\author{
Nobuaki Ochi',2 \\ Hiromichi Yamane ${ }^{1}$ \\ Katsuyuki Hotta ${ }^{3}$ \\ Hiromi Fujii ${ }^{4}$ \\ Hideko Isozaki'2,5 \\ Yoshihiro Honda ${ }^{1,2}$ \\ Tomoko Yamagishi' \\ Toshio Kubo ${ }^{6}$ \\ Mitsune Tanimoto ${ }^{2,3}$ \\ Katsuyuki Kiura ${ }^{6}$ \\ Nagio Takigawa'
}

'Department of General Internal Medicine 4, Kawasaki Hospital, Kawasaki Medical School, Okayama, Japan; ${ }^{2}$ Department of Hematology,

Oncology and Respiratory Medicine, Okayama University Graduate School of Medicine, Dentistry, and Pharmaceutical Sciences, Okayama, Japan; ${ }^{3}$ Department of Hematology and Oncology, Okayama University Hospital, Okayama, Japan; ${ }^{4}$ Department of Pharmacy, Kawasaki Hospital, Kawasaki Medical School, Okayama, Japan; ${ }^{5}$ Department of Clinical Pharmaceutics, Okayama University Graduate School of Medicine, Dentistry, and Pharmaceutical Sciences, Okayama, Japan; ${ }^{6}$ Department of Respiratory Medicine, Okayama University Hospital, Okayama, Japan

Correspondence: Nagio Takigawa Department of General Internal Medicine 4, Kawasaki Hospital, Kawasaki Medical School, 2-I-80 Nakasange,

Kita-ku, Okayama, Japan

$\mathrm{Tel}+8$ I 862252 I I I

Fax +8I 862328343

Email ntakigaw@gmail.com
Introduction: Widespread use of generic drugs is considered to be indispensable if reductions in total health care costs are to be achieved, but the market share of such drugs remains low. In general, generic drugs have the same active ingredients as brand-name drugs, but this is not always the case. Thus, toxicity profiles may vary when brand-name and generic drugs are compared. We retrospectively investigated the incidence of hyponatremia in patients receiving brand-name cisplatin (CDDP) and a generic counterpart thereof.

Methods: We reviewed the medical records of patients treated with brand-name CDDP ( $\mathrm{n}=53$ ) and a generic formulation $(\mathrm{n}=26)$, and compared the incidences of hyponatremia and renal toxicity. Toxicities were graded using the Common Terminology Criteria for Adverse Events, version 4.0. Differences between groups were evaluated using the Student's $t$-test, and the odds ratio for hyponatremia was estimated via logistic regression analysis.

Results: Serum creatinine levels after chemotherapy increased significantly in both the brandname and generic CDDP groups; no significant difference was evident between the two groups. Hyponatremia of grade 3 or above developed in $30.7 \%$ of the generic CDDP group compared to $15.1 \%$ of the brand-name CDDP group $(P=0.011)$. Multivariate analysis showed that the use of generic CDDP increased the incidence of hyponatremia (odds ratio $=5.661,95 \%$ confidence interval $=1.403-22.839 ; P=0.015)$.

Conclusion: Oncologists should be aware that use of a generic CDDP might be associated with more hyponatremia than would use of brand-name CDDP.

Keywords: cisplatin, hyponatremia, brand-name, generic drug

\section{Introduction}

Generic drugs containing the same active ingredients as the original brand-name products have been developed and prescribed worldwide. Widespread use of such drugs is considered to be indispensable to reduce health care costs. ${ }^{1}$ However, the market share of generic drugs is not yet sufficiently large to notably decrease the total health care expenditure in many developed countries. One reason is that both patients and physicians are unsure that the effects and safety profiles of generic drugs are truly similar to those of brand-name drugs. As generics contain the same active ingredients as brand-name drugs, the bioavailability of a generic is generally assumed to be the same as that of the brand-name drug, and no additional clinical trials are performed.

Cisplatin (CDDP) is one of the most active antineoplastic drugs prescribed to treat various solid tumors, including lung, gastric, head and neck, and bladder cancers. CDDP remains the principal drug of choice for treatment of these malignancies despite the development of many novel anticancer drugs over the past several decades. ${ }^{2,3}$ However, CDDP induces many adverse effects, including nausea, vomiting, appetite loss, renal 
toxicity, and neurotoxicity. The "syndrome of inappropriate secretion of antidiuretic hormone" (SIADH) is a less common but important toxicity that is sometimes fatal. CDDP-induced hyponatremia has also occurred in a case of renal salt-wasting syndrome, ${ }^{4}$ in patients with nausea/vomiting/anorexia, and when vigorous hydration was administered to prevent nephrotoxicity. ${ }^{5}$ It is sometimes difficult to accurately diagnose the cause of hyponatremia because various contributing factors can interact in a complex manner.

To date, a few reports have explored differences in the renal toxicities of brand-name and generic CDDP. ${ }^{6,7}$ Here, we focused on the frequency of hyponatremia induced by a generic CDDP compared with its brand-name counterpart.

\section{Materials and methods}

\section{Patient selection}

We searched for patients receiving CDDP-containing regimens from January 2009 to April 2010 at Okayama University Hospital and from April 2011 to March 2013 at Kawasaki Hospital, both in Okayama, Japan. Over these respective periods, the two hospitals prescribed both brand-name and generic CDDP. Patients were retrospectively selected using the following criteria: 1) histologically or cytologically diagnosed malignancy; 2) no prior chemotherapy with a CDDP-containing regimen; 3) chemotherapy with a regimen that included more than $60 \mathrm{mg}$ of CDDP on day 1; and 4) a baseline sodium concentration $>130 \mathrm{mEq} / \mathrm{L}$. Patients receiving concurrent radiation therapy were excluded. Both groups received their CDDP-containing treatments in the context of a short-duration hydration setting. ${ }^{8}$ To avoid interactions between hydration settings, we extracted additional patients who received treatment with a brand-name CDDP-containing regimen under standard hydration conditions (approximately 3,000 $\mathrm{mL}$ on day 1) from September 2007 to December 2008 in Okayama University Hospital. We selected this period because NO and NT, who hold board certificates for medical oncology, worked at Okayama University Hospital at that time. We could treat patients with only brandname CDDP at Okayama University, and with only generic CDDP at Kawasaki Hospital in the respective periods because of availability of the drugs. This study was approved by the Kawasaki Medical School Ethics Committee (\# 1636) and Okayama University Hospital Ethics Committee (\# 1889).

\section{Data collection process and data items}

To avoid bias in data abstraction, four medical staff(YH, HF, HI, and NO) independently abstracted data from electronic medical records and subsequently compared their results. The following information was obtained from each report: date of treatment administration; treatment regimen; dose of CDDP; and patient characteristics (age, sex, underlying malignancy, performance status [PS], duration of hyponatremia, pretreatment and posttreatment serum sodium concentrations, serum creatinine level, serum potassium level, and body surface area). Serum biochemical levels were measured using automatic analyzers (Biomajesty ${ }^{\mathrm{TM}} \mathrm{JCA}-\mathrm{BM}$ series; JEOL, Tokyo, Japan) in both institutes.

\section{Treatment schedule}

Patients treated with brand-name and generic CDDP under short-duration hydration conditions ${ }^{8}$ were classified into groups 1 and 3, respectively, and patients treated with brand-name CDDP using standard hydration were defined as group 2. Briefly, the short hydration regimen for groups 1 and 3 was as follows. Antiemetic prophylaxis consisted of a 5-hydroxytryptamine $3\left(5-\mathrm{HT}_{3}\right)$ receptor antagonist and dexamethasone, with or without an aprepitant, and was given before chemotherapy. For prehydration and posthydration, $5 \mathrm{mEq}$ potassium chloride and $4 \mathrm{mEq}$ magnesium sulfate in $500 \mathrm{~mL}$ of half saline solution were infused at a rate of $500 \mathrm{~mL} /$ hour. Next, cytotoxic agents (docetaxel, pemetrexed, irinotecan, vinorelbine, gemcitabine, etoposide, or topotecan), in 100-250 $\mathrm{mL}$ of normal saline, were administered, followed by $40 \mathrm{~g}$ mannitol (to trigger diuresis) 15 minutes later, and an appropriate dose of CDDP in $250 \mathrm{~mL}$ of normal saline 1 hour later. At least $1,000 \mathrm{~mL}$ of water was taken orally on days 2 and 3; this was mandatory because traditional intravenous hydration was not performed.

\section{Definition of hyponatremia}

Hyponatremia was defined and graded using the Common Terminology Criteria for Adverse Events, version 4.0, as follows: a serum sodium concentration from lower limit of normal to $130 \mathrm{mEq} / \mathrm{L}$, grade $1 ;<130-120 \mathrm{mEq} / \mathrm{L}$, grade $3 ;<120 \mathrm{mEq} / \mathrm{L}$, grade 4 ; and death from hyponatremia, grade 5 . We graded all patients using these criteria. We collected laboratory data and information on patient characteristics from electronic medical databases.

\section{Statistical analysis}

Differences between the two groups were evaluated using Student's $t$-tests (paired and unpaired) to compare parametric data and the Wilcoxon signed-rank test or Mann-Whitney $U$-test to compare nonparametric data. Differences between groups were evaluated via one-way analysis of variance. The Bonferroni test was applied when multiple comparisons were performed. Relationships between serum sodium concentrations and patient characteristics were analyzed via linear regression. Odds ratios with $95 \%$ confidence intervals 
(CIs) for development of hyponatremia, by strata of patient characteristics, were estimated via logistic regression. Such analyses were weighted by the delta serum sodium concentration $(\Delta \mathrm{Na})$, defined as the difference between the prechemotherapy serum sodium concentration and the minimum sodium concentration. A positive $\Delta \mathrm{Na}(>0)$ meant that the serum sodium concentration decreased during treatment. All $P$-values were obtained by two-sided testing, and the significance level was set at $P<0.05$. Statistical analyses were conducted using the Stata software (version 12; StataCorp, College Station, TX, USA).

\section{Results}

A total of 79 patients met the study criteria, of whom 53 received brand-name CDDP, 27 via short hydration (group 1) and 26 via standard hydration (group 2). A total of 26 patients were treated with generic CDDP via short hydration (group 3). Patient characteristics are shown in Table 1. No significant difference in any of age, PS, the number of drug regimens given, or baseline serum sodium concentration was evident between groups 1 and 3 . The proportion of male patients in group 1 (88.9\%) was significantly higher than in group 3 (53.8\%).

Hyponatremia status and serum creatinine levels after chemotherapy are shown in Table 2. No treatment-related death was noted. Grade $3(19.2 \%)$ and grade 4 (11.5\%) hyponatremia were observed in group 3 , whereas grade 4 hyponatremia was not detected in groups 1 or 2 . The $\Delta \mathrm{Na}$ value $(7.5 \mathrm{mEq} / \mathrm{L})$ in group 3 was marginally higher than that $(6.0 \mathrm{mEq} / \mathrm{L})$ in group $1(P=0.05)$. Hyponatremia of grade 3 or above occurred in $30.7 \%$ of the generic CDDP group (group 3) compared to $15.1 \%$ of the brand-name CDDP group (groups 1 and 2$)(P=0.011)$. The mean minimum sodium concentrations were $129.5 \mathrm{mEq} / \mathrm{L}$ in the generic CDDP group and $132.7 \mathrm{mEq} / \mathrm{L}$ in the brand-name CDDP group (Figure 1A). When the three groups were compared, the sodium concentration in group 3 was significantly lower than in group $1(P=0.034)$. However, no significant difference was evident between groups 1 and 2 (Figure 1B). These results suggest that hyponatremia might be induced not by short hydration but rather by use of generic CDDP.

Table I Patient characteristics

\begin{tabular}{|c|c|c|c|c|c|}
\hline Characteristic & Group I $(n=27)$ & Group $2(n=26)$ & Group $3(n=26)$ & Total $(n=79)$ & $P$-value* \\
\hline \multicolumn{6}{|l|}{ Age (years) } \\
\hline Mean & 62.8 & 58.3 & 63.8 & 61.6 & 0.67 \\
\hline Range & $35.1-78.5$ & $33.0-76.7$ & $37.1-74.6$ & $33.0-78.5$ & \\
\hline \multicolumn{6}{|l|}{ Performance status (\%) } \\
\hline$\geq 1$ & 81.5 & 61.5 & 80.8 & 74.7 & 0.63 \\
\hline Sex, male $(\%)$ & 88.9 & 80.8 & 53.8 & 69.6 & $<0.0 \mathrm{I}^{\dagger}$ \\
\hline \multicolumn{6}{|l|}{ Treatment line } \\
\hline Median & 1 & 1 & 1 & 1 & 0.04 \\
\hline Range & $1-6$ & $1-6$ & $1-9$ & $1-9$ & \\
\hline \multicolumn{6}{|c|}{ Serum sodium concentration (mEq/L) } \\
\hline Median & 139 & 139 & 139 & 139 & 0.37 \\
\hline Range & $130-143$ & $136-144$ & $|33-14|$ & $130-144$ & \\
\hline \multicolumn{6}{|c|}{ Serum potassium concentration (mEq/L) } \\
\hline Median & 4.10 & 4.20 & 4.25 & 4.20 & 0.48 \\
\hline Range & $3.6-5.1$ & $3.8-4.9$ & $3.2-5.4$ & $3.2-5.4$ & \\
\hline \multicolumn{6}{|c|}{ Serum creatinine level (mg/dL) } \\
\hline Median & 0.79 & 0.75 & 0.63 & 0.72 & $<0.05^{\dagger}$ \\
\hline Range & $0.5-1.07$ & $0.44-1.10$ & $0.44-1.18$ & $0.44-1.18$ & \\
\hline \multicolumn{6}{|l|}{ Cisplatin dose $\left(\mathrm{mg} / \mathrm{m}^{2}\right)$} \\
\hline Mean & 65.8 & 67.6 & 71.5 & 68.2 & $<0.05^{\dagger}$ \\
\hline Range & $54.5-80.1$ & $53.6-83.4$ & $55.4-84.7$ & $53.6-84.7$ & \\
\hline \multicolumn{6}{|l|}{ Type of malignancy } \\
\hline NSCLC & 24 & 21 & 17 & 79 & \\
\hline Adenocarcinoma & 17 & 14 & 12 & 43 & \\
\hline Squamous cell cancer & 3 & 6 & 4 & 13 & \\
\hline LCNEC & 3 & 1 & 0 & 4 & \\
\hline Other & I & 0 & 1 & 2 & \\
\hline SCLC & 3 & 5 & I & 9 & \\
\hline Digestive organ & 0 & 0 & 6 & 6 & \\
\hline Others & 0 & 0 & 2 & 2 & \\
\hline
\end{tabular}

Notes: $* P$-values were assessed between groups I and 3 by two-sample Wilcoxon rank sum test or unpaired Student's $t$-test. ${ }^{\prime}$ Statistically significant at $P<0.05$. Abbreviations: LCNEC, large-cell neuroendocrine carcinoma; NSCLC, non-small-cell lung cancer; SCLC, small-cell lung cancer. 
Table 2 Hyponatremic status and serum creatinine levels after chemotherapy

\begin{tabular}{|c|c|c|c|c|c|}
\hline Variable & Group I $(n=27)$ & Group $2(n=26)$ & Group $3(n=26)$ & Total $(n=79)$ & $P$-value* \\
\hline \multicolumn{6}{|c|}{ Minimum serum sodium concentration (mEq/L) } \\
\hline Median & 134.0 & 132.5 & 130.5 & 133.0 & $<0.05^{\dagger}$ \\
\hline Range & $122-139$ & $12 \mid-138$ & $112-138$ & $112-139$ & \\
\hline \multicolumn{6}{|c|}{ Hyponatremia (\%) } \\
\hline Grade I & 44.4 & 57.7 & 50.0 & 50.6 & \\
\hline Grade 3 & 14.8 & 15.4 & 19.2 & 16.5 & \\
\hline Grade 4 & 0.0 & 0.0 & 11.5 & 3.8 & \\
\hline \multicolumn{6}{|c|}{$\Delta \mathrm{Na}(\mathrm{mEq} / \mathrm{L})$} \\
\hline Median & 6.0 & 6.5 & 7.5 & 6.0 & $0.05^{\dagger}$ \\
\hline Range & $1-19$ & $2-22$ & $\mathrm{I}-28$ & $\mathrm{I}-28$ & \\
\hline \multicolumn{6}{|c|}{ Duration (day) } \\
\hline Median & 6.0 & 7.0 & 7.8 & 7.0 & $0.4 I$ \\
\hline Range & $3-33$ & $4-46$ & $3-33$ & $3-46$ & \\
\hline \multicolumn{6}{|c|}{ Post-serum creatinine level (mg/dL) } \\
\hline Median & 0.86 & 0.97 & 0.68 & 0.89 & 0.44 \\
\hline Range & $0.5 \mathrm{I}-1.76$ & $0.63-1.66$ & $0.45-1.75$ & $0.45-1.76$ & \\
\hline
\end{tabular}

Notes: $* P$-values were assessed between groups I and 3 by unpaired Student's $t$-test. 'Statistically significant at $P<0.05$.

Abbreviation: $\Delta \mathrm{Na}$, difference between the prechemotherapy serum sodium concentration and the minimum sodium concentration.

Serum creatinine concentrations after chemotherapy (the maximum values attained during chemotherapy) increased uniformly, with statistical significance, in all groups (Figure 2A). Baseline serum creatinine levels were slightly but significantly higher in the brand-name CDDP group compared to the generic CDDP group $(P=0.0124)$; however, no significant between-group difference was evident in serum creatinine levels after chemotherapy (Figure 2B). To exclude any effect of hydration, we focused on serum creatinine changes in groups 1 and 3. The increases in serum creatinine levels did not differ between these groups (Figure 2C), indicating that the extents of renal toxicity were similar, regardless of the CDDP formulation used. Also, sex did not affect the results (Figure 2D).
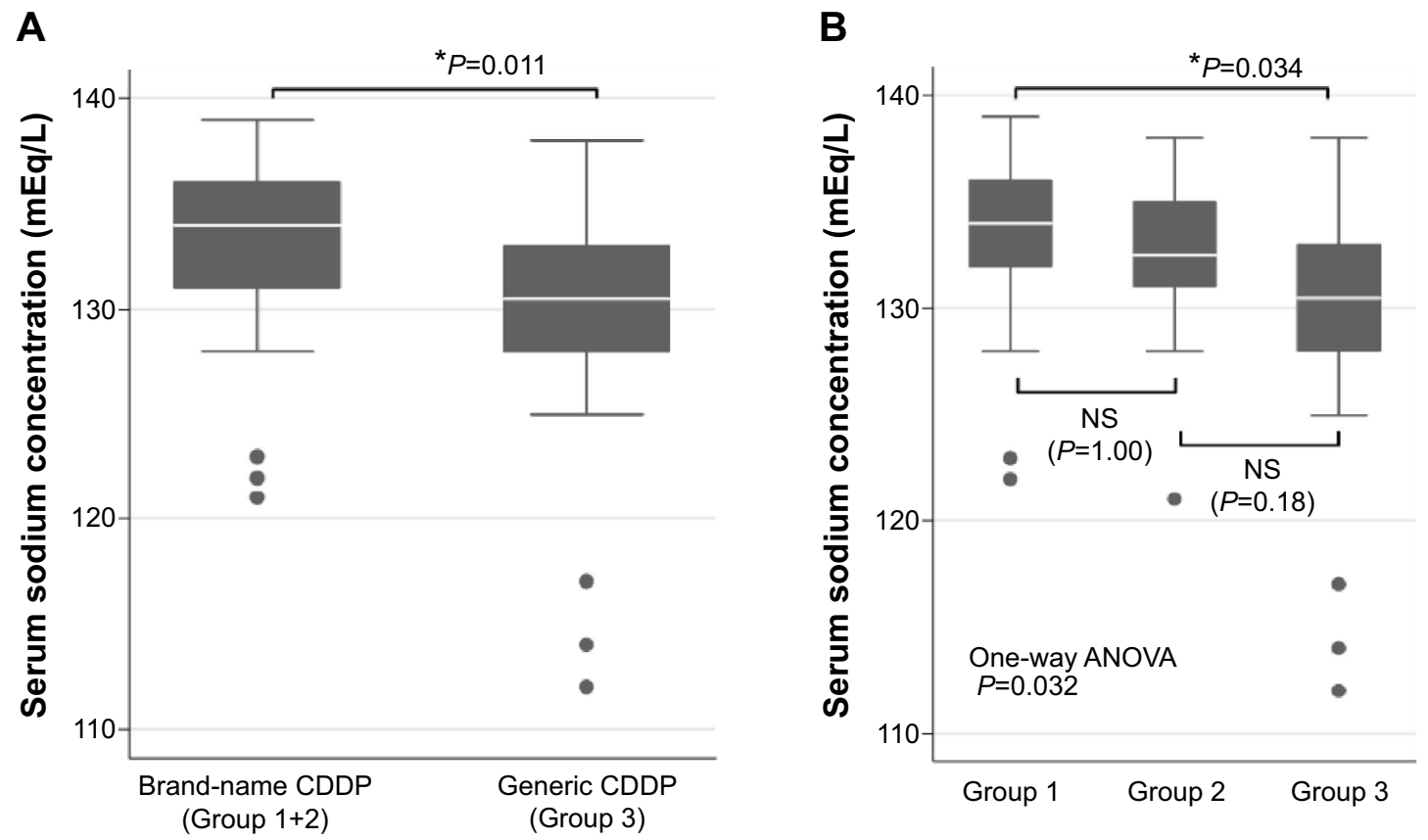

Figure I Minimum serum sodium concentrations during chemotherapy.

Notes: Box-and-whisker plots. The bottom and top of each box are the 25 th and 75 th percentiles (the lower and upper quartiles, respectively), and the band near the middle of each box is the 50th percentile (the median). Comparisons of minimum serum sodium concentrations (A) between those receiving brand-name CDDP (groups I and 2 ) and generic CDDP (group 3) and (B) among all three groups. *Significant at $P<0.05$.

Abbreviations: ANOVA, analysis of variance; CDDP, cisplatin; NS, not significant. 

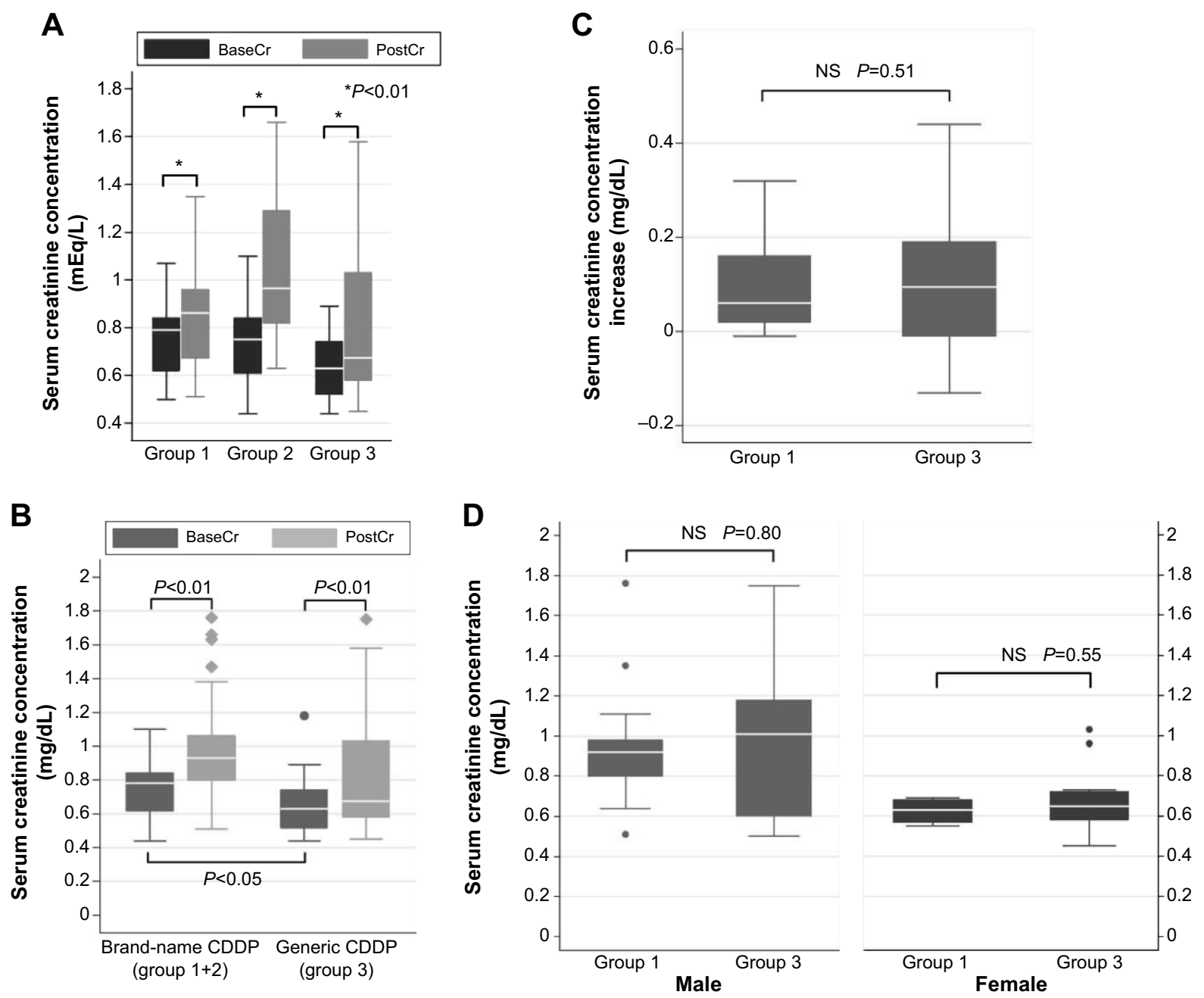

Figure 2 Serum creatinine concentrations before and after chemotherapy.

Notes: Box-and-whisker plots. The bottom and top of each box are the 25 th and 75 th percentiles (the lower and upper quartiles, respectively), and the band near the middle of each box is the 50th percentile (the median). Comparisons of serum creatinine concentrations (A) before chemotherapy (BaseCr) and after chemotherapy (PostCr) in each group, or (B) between those receiving brand-name CDDP (groups I and 2) and generic CDDP (group 3). (C) Comparison of the differences in serum creatinine concentrations before and after chemotherapy in groups I and 3. (D) Comparison of the serum creatinine concentrations of groups I and 3 patients, by sex.

Abbreviations: BaseCr, serum creatinine concentration before chemotherapy; CDDP, cisplatin; PostCr, serum creatinine concentration after chemotherapy; NS, not significant.

Linear regression analyses revealed no significant influence of any patient characteristic (age, baseline serum creatinine level, or CDDP dose) on serum sodium concentration in the two groups (Figure 3). The serum sodium concentration tended to decrease in patients with low baseline sodium concentrations (the regression coefficient was $0.73 ; P=0.051)$, indicating that a patient lost $0.73 \mathrm{mEq} / \mathrm{L}$ serum sodium upon brand-name CDDP treatment with each decrement of $1.0 \mathrm{mEq} / \mathrm{L}$ at a baseline serum sodium concentration. In other words, $\Delta \mathrm{Na}$ values in patients receiving brand-name CDDP seemed to remain constant irrespective of baseline sodium concentrations, whereas use of generic CDDP seemed to unexpectedly decrease the sodium concentration regardless of the baseline concentration (Figure 3B).
We further explored key factors influencing development of grade 3 or 4 hyponatremia. All variables were dichotomized into two classes, on the basis of median or mean values. Univariate and multivariate analyses of several variables are shown in Table 3. Generic CDDP use was associated with a trend toward hyponatremia, but without statistical significance (odds ratio $=2.888,95 \%$ CI $=0.837-9.966$; $P=0.093$ ), upon univariate analysis. Although patients with low baseline serum sodium concentrations $(<139 \mathrm{mEq} / \mathrm{L})$ developed grade 3 or 4 hyponatremia more frequently, this was not statistically significant (odds ratio $=2.348,95 \%$ $\mathrm{CI}=0.701-7.860 ; P=0.166$ ). Multivariate analysis revealed that generic CDDP use was associated with a 5.661-fold increased risk of hyponatremia $(95 \% \mathrm{CI}=1.403-22.839$; $P=0.015$ ). Also, old age ( $\geq 70$ years) and poor PS ( 1 or 

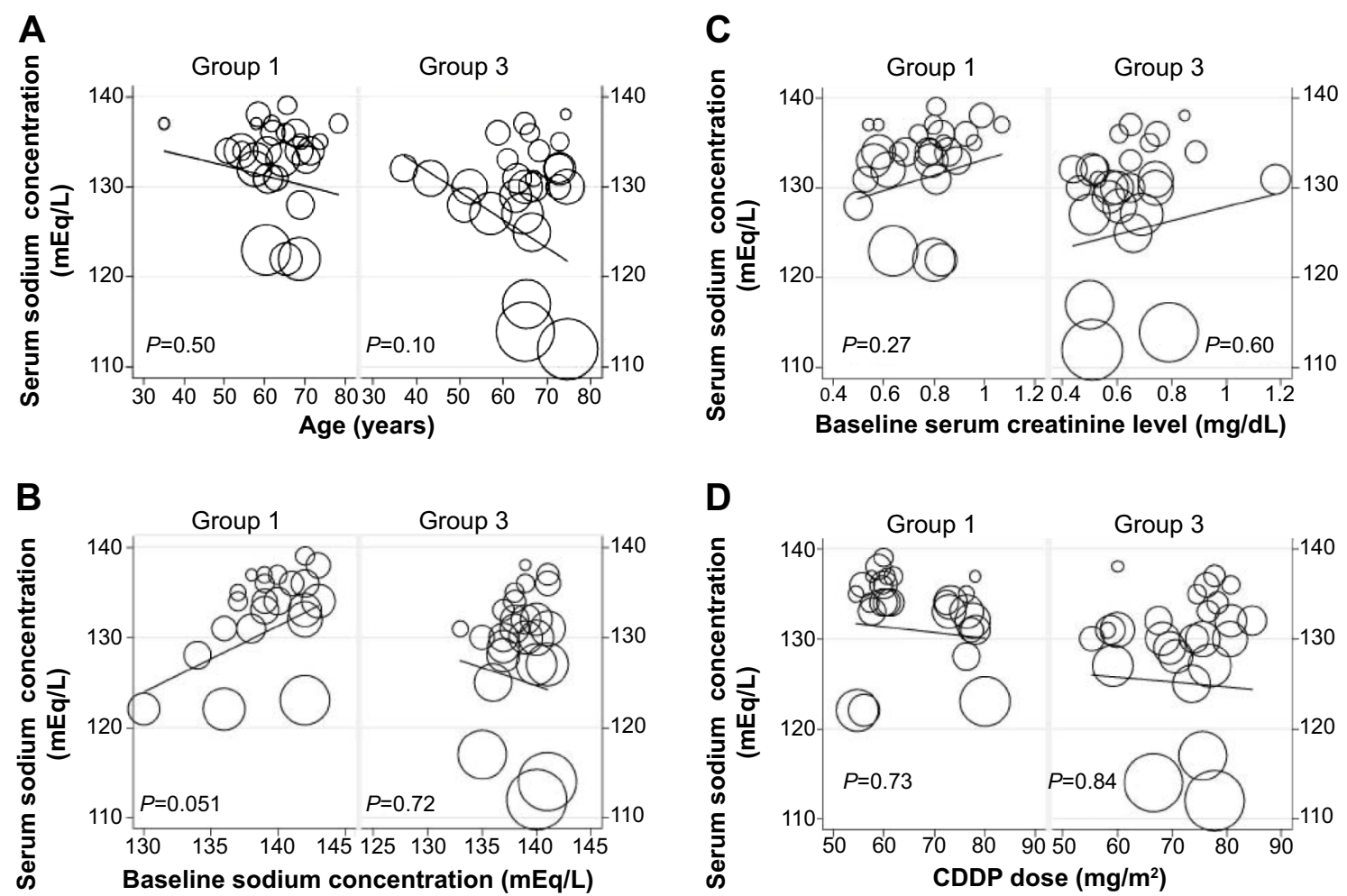

Figure 3 Relationships between serum sodium concentrations and patient characteristics.

Notes: Associations between the minimum serum sodium concentrations after chemotherapy and (A) age, (B) baseline serum creatinine concentration, (C) baseline serum sodium concentration, and (D) CDDP dose, in groups I and 3 .

Abbreviation: CDDP, cisplatin.

above) were associated with increased risks of hyponatremia, but such associations were statistically of marginal significance.

\section{Discussion}

We found that the incidence of hyponatremia increased significantly in patients treated with a generic CDDP rather than brand-name CDDP. In contrast, the incidence of renal damage was similar in the two groups. Use of a generic CDDP significantly increased the frequency of grade 3 or 4 hyponatremia (odds ratio $=5.661$ ).
The question of whether generic drugs are truly equivalent to brand-name drugs has often been debated. When an application for release of a brand-name drug is made, the US Food and Drug Administration (FDA) requires drug manufacturers to submit large amounts of preclinical and clinical data on safety and efficacy and, in most cases, requires clinical data from large-scale trials conducted in the intended patient populations. However, an application for release of a generic drug need not be accompanied by the same amount of clinical safety and efficacy data; it is necessary only to demonstrate that the bioavailability and bioequivalence are equivalent to those of

Table 3 Univariate and multivariate analysis of variables

\begin{tabular}{|c|c|c|c|c|c|c|}
\hline \multirow[t]{2}{*}{ Variable } & \multicolumn{3}{|c|}{ Univariate analysis } & \multicolumn{3}{|c|}{ Multivariate analysis* } \\
\hline & Odds ratio & $P$-value & $95 \% \mathrm{Cl}$ & Odds ratio & P-value & $95 \% \mathrm{Cl}$ \\
\hline Male & 0.355 & 0.103 & $0.102-1.234$ & & $0.181^{\dagger}$ & \\
\hline Age $\geq 70$ years & 1.505 & 0.713 & $0.171-13.267$ & 6.756 & 0.068 & $0.868-52.608$ \\
\hline$P S \geq I$ & 0.624 & 0.493 & $0.162-2.400$ & 0.231 & 0.066 & $0.048-1.104$ \\
\hline Generic CDDP & 2.888 & 0.093 & $0.837-9.966$ & 5.661 & 0.015 & I.403-22.839 \\
\hline Short hydration & 1.855 & 0.375 & $0.474-7.265$ & & $0.360^{\dagger}$ & \\
\hline Baseline sodium concentration $<139 \mathrm{mEq} / \mathrm{L}$ & 2.348 & 0.166 & $0.70 \mathrm{I}-7.860$ & & $0.24 \mathrm{I}^{\dagger}$ & \\
\hline Baseline creatinine concentration $\geq 0.72 \mathrm{mg} / \mathrm{dL}$ & 0.619 & 0.451 & $0.178-2.155$ & & $0.806^{\dagger}$ & \\
\hline CDDP dose $\geq 70 \mathrm{mg} / \mathrm{m}^{2}$ & 0.674 & 0.526 & $0.198-2.287$ & & $0.378^{\dagger}$ & \\
\hline
\end{tabular}

Notes: *Stepwise multiple regression analysis. †Variable was removed because of no significance for analysis.

Abbreviations: $\mathrm{Cl}$, confidence interval; CDDP, cisplatin; PS, performance status. 
the brand-name drug. ${ }^{9}$ Furthermore, the FDA generally allows generic drug peak plasma concentration, and the area under the plasma drug concentration versus time curve (the AUC), to vary by $-20 \%$ to $+25 \%$ compared to the brand-name drug. ${ }^{9}$ It is possible that such variation may render the efficacy and safety of generic drugs different from those of their brand-name counterparts. Any increase in toxicity or reduction in efficacy upon slight dose escalation or reduction is of concern, because the dosages of anticancer drugs are finely adjusted by reference to the body surface area or body weight of patients.

A large-scale retrospective analysis of the bioequivalence data on generic and brand-name drugs, accumulated over a 12-year period, showed that the average differences in peak plasma concentration and AUC values between generic and brand-name products were $4.35 \%$ and $3.56 \%$, respectively. ${ }^{10}$ It was concluded that generic drugs were therapeutically equivalent to their brand-name counterparts. However, when differences between brand-name drugs and their generic counterparts used in the fields of cardioangiology, ${ }^{11-13}$ psychiatry, ${ }^{14-17}$ and endocrinology ${ }^{18-20}$ were compared, the results were controversial.

Possible differences between brand-name and generic drugs delivered via injection have received less attention. ,, $721-27^{-1}$ Generic and brand-name drugs contain the same active ingredients and exhibit similar pharmacokinetics. However, the drugs may differ in terms of safety and toxicity profiles because some of the inactive ingredients vary. Most generic formulations of docetaxel contained a lower drug concentration than the brand-name formulation (less than $90 \%$ of that expected in some instances) and/or high levels of impurities absent from the brand-name drug. ${ }^{22}$ In vivo studies have shown that the blood levels of some liver enzymes increased in those taking generic paclitaxel but not the brandname drug, but no significant differences in neurotoxicity, hypersensitivity, or vascular pain were evident. ${ }^{25,28}$

As is also true of other antineoplastic drugs, CDDP-induced toxicity has been mentioned frequently in the literature, but few reports have explored differences between generic and brandname formulations. Recently, differences in renal toxicities have been reported. Sekine et $\mathrm{al}^{6}$ found that grade 2 or 3 renal toxicity was more frequently observed in male patients taking the generic formulation, because their hydration volumes were relatively low. Another large-scale retrospective study found that grade 1 renal toxicity was significantly more frequent in the generic CDDP group, but the incidences of grade 2 or 3 toxicities did not differ between those taking the two CDDP formulations. ${ }^{7}$ In our present study, we found no significant among-group difference in renal toxicity. Differences in the hematological toxicities of brand-name and generic CDDP have also been investigated retrospectively. In patients with cervical cancer, the number experiencing grade 3 or 4 leukopenia was significantly greater in the generic than in the brandname group. ${ }^{27} \mathrm{We}$ focused on renal toxicity and hyponatremia in the present study, but other toxicities - including hematological toxicity - should be investigated in future studies. Like docetaxel described above, generic cisplatin might have some impurities that the brand-name cisplatin does not have. It is very important to find out whether the brand-name cisplatin has better quality control and whether any impurities have interfered with the therapy. A further study including heavy-metal analysis should be performed in order to clarify this.

Our present study had several limitations. First, this was a small retrospective study; thus, a larger randomized study should be performed to confirm our results. Realistically, a prospective observational study might be preferable. Second, because we included all consecutive patients who were eligible, all of the nature of underlying malignancies, the extent of hyponatremia before chemotherapy, and the chemotherapeutic drug combinations prescribed differed slightly among the groups. The other combined agent and some of the diseases, such as brain tumors, could account for hyponatremia, including SIADH. It is preferable to unify the chemotherapeutic regimen, underlying malignancies, and disease stages in any further observational study, as our results may have been affected by such differences. Third, we had the data such as urinary sodium concentration and osmolality only in several patients. Thus, the causes of hyponatremia were not extensively examined. SIADH, renal salt-wasting syndrome, and salt loss caused by vomiting and/or anorexia, should be diagnosed as accurately as possible in future studies.

In conclusion, generic CDDP may cause hyponatremia more frequently than the brand-name drug because of slight differences in active or inactive ingredients. Generic drugs have been widely used worldwide and contribute to reductions in health care costs. However, clinicians should be aware that the use of generic drugs often requires a trade-off between cost-effectiveness and assured safety, because off-patent drugs are generally approved for sale without the performance of further expensive and time-consuming clinical trials.

\section{Acknowledgement}

We thank Dr Yasumasa Monobe, Department of Pathology 1, Kawasaki Hospital, Kawasaki Medical School, Okayama, Japan, who confirmed the diagnoses of malignancies.

\section{Disclosure}

$\mathrm{KH}$ has received honoraria from Eli Lilly Japan, Taiho Pharmaceutical, Chugai Pharmaceutical, and Sanofi-Aventis. 
KK has received honoraria from Eli Lilly Japan, Nihon Kayaku, AstraZeneca, Daiichi-Sankyo Pharmaceutical, Chugai Pharmaceutical, Taiho Pharmaceutical, and SanofiAventis. NT has received honoraria from Eli Lilly Japan, Nihon Kayaku, AstraZeneca, Daiichi-Sankyo Pharmaceutical, Chugai Pharmaceutical, Taiho Pharmaceutical, Pfizer Inc. Japan, Boehringer-Ingelheim in Japan, and SanofiAventis. The authors report no other conflicts of interest in this work.

\section{References}

1. Hartman M, Martin A, McDonnell P, Catlin A; National Health Expenditure Accounts Team. National health spending in 2007: slower drug spending contributes to lowest rate of overall growth since 1998. Health Aff (Millwood). 2009;28(1):246-261.

2. Hotta K, Matsuo K, Ueoka H, Kiura K, Tabata M, Tanimoto M. Metaanalysis of randomized clinical trials comparing Cisplatin to Carboplatin in patients with advanced non-small-cell lung cancer. J Clin Oncol. 2004;22(19):3852-3859.

3. Hotta K, Matsuo K. Long-standing debate on cisplatin- versus carboplatin-based chemotherapy in the treatment of advanced non-small cell lung cancer. J Thorac Oncol. 2007;2(1):96.

4. Hamdi T, Latta S, Jallad B, KheirF, Alhosaini MN, Patel A. Cisplatin-induced renal salt wasting syndrome. South Med J. 2010;103(8):793-799.

5. Raftopoulos H. Diagnosis and management of hyponatremia in cancer patients. Support Care Cancer. 2007;15(12):1341-1347.

6. Sekine I, Kubota K, Tamura Y, et al. Innovator and generic cisplatin formulations: comparison of renal toxicity. Cancer Sci. 2011;102(1):162-165.

7. Niho $\mathrm{S}$, Yamanaka T, Umemura S, et al. Renal toxicity caused by brandname versus generic cisplatin: a comparative analysis. Jpn J Clin Oncol. 2013;43(4):390-395.

8. Hotta K, Takigawa N, Hisamoto-Sato A, et al. Reappraisal of short-term low-volume hydration in cisplatin-based chemotherapy: results of a prospective feasibility study in advanced lung cancer in the Okayama Lung Cancer Study Group Trial 1002. Jpn J Clin Oncol. 2013;43(11): $1115-1123$.

9. Kefalas $\mathrm{CH}$, Ciociola AA. The FDA's generic-drug approval process: similarities to and differences from brand-name drugs. Am J Gastroenterol. 2011;106(6):1018-1021.

10. van der Meersch A, Dechartres A, Ravaud P. Quality of reporting of bioequivalence trials comparing generic to brand name drugs: a methodological systematic review. PLoS One. 2011;6(8):e23611.

11. Kesselheim AS, Misono AS, Lee JL, et al. Clinical equivalence of generic and brand-name drugs used in cardiovascular disease: a systematic review and meta-analysis. JAMA. 2008;300(21):2514-2526.

12. Dentali F, Donadini MP, Clark N, et al; Warfarin Associated Research Projects and Other Endeavors (WARPED) Consortium. Brand name versus generic warfarin: a systematic review of the literature. Pharmacotherapy. 2011;31(4):386-393.

13. Caldeira D, Fernandes RM, Costa J, David C, Sampaio C, Ferreira JJ. Branded versus generic clopidogrel in cardiovascular diseases: a systematic review. J Cardiovasc Pharmacol. 2013;61(4):277-282.
14. Kesselheim AS, Stedman MR, Bubrick EJ, et al. Seizure outcomes following the use of generic versus brand-name antiepileptic drugs: a systematic review and meta-analysis. Drugs. 2010;70(5):605-621.

15. Yamada M, Welty TE. Generic substitution of antiepileptic drugs: a systematic review of prospective and retrospective studies. Ann Pharmacother. 2011;45(11):1406-1415.

16. Emanuele E. Generic versus brand name drugs in psychopharmacology: a pharmacoeconomic perspective. South Med J. 2011;104(10): 715-716.

17. Talati R, Scholle JM, Phung OP, et al. Efficacy and safety of innovator versus generic drugs in patients with epilepsy: a systematic review. Pharmacotherapy. 2012;32(4):314-322.

18. Dong BJ, Hauck WW, Gambertoglio JG, et al. Bioequivalence of generic and brand-name levothyroxine products in the treatment of hypothyroidism. JAMA. 1997;277(15):1205-1213.

19. Kim SH, Park K, Hong SJ, et al. Efficacy and tolerability of a generic and a branded formulation of atorvastatin $20 \mathrm{mg} / \mathrm{d}$ in hypercholesterolemic Korean adults at high risk for cardiovascular disease: a multicenter, prospective, randomized, double-blind, double-dummy clinical trial. Clin Ther. 2010;32(11):1896-1905.

20. Carswell JM, Gordon JH, Popovsky E, Hale A, Brown RS. Generic and brand-name L-thyroxine are not bioequivalent for children with severe congenital hypothyroidism. J Clin Endocrinol Metab. 2013;98(2): 610-617.

21. Olufolabi AJ, Gan TJ, Lacassie HJ, White WD, Habib AS. A randomized, prospective double-blind comparison of the efficacy of generic propofol (sulphite additive) with diprivan. Eur J Anaesthesiol. 2006; 23(4):341-345.

22. Vial J, Cohen M, Sassiat P, Thiébaut D. Pharmaceutical quality of docetaxel generics versus originator drug product: a comparative analysis. Curr Med Res Opin. 2008;24(7):2019-2033.

23. Leelarasamee A, Rongrungruang Y, Trakulsomboon S, Pongpech P, Thanawattanawanich $P$, Jithavech $P$. Bioequivalence, antibacterial activity and therapeutic outcome of a generic meropenem (Mapenem). J Med Assoc Thai. 2008;91(7):980-988.

24. Thamlikitkul V, Tiengrim S, Chalermsri C, Chinsawangwatanakul P, Suddhichupaiboon S. Microbiological equivalence of serum bacteriostatic and bactericidal activities of the sera from healthy volunteers receiving original meropenem (Meronem) and generic meropenem (Mero). J Med Assoc Thai. 2010;93 Suppl 1:S110-S116.

25. Nakamura I, Ichimura E, Kobayashi H, et al. Comparative study of peripheral neurotoxicity after injection of two different paclitaxel formulations in rats. Arzneimittelforschung. 2010;60(4):205-209.

26. Thamlikitkul V, Tiengrim S, Chalermsri C, Sae-Jong R, Suddhichupaiboon S. Microbiological equivalence of bacteriostatic and bactericidal activities of the sera from healthy volunteers receiving generic piperacillin/tazobactam (Pipertaz) and original piperacillin/tazobactam (Tazocin). J Med Assoc Thai. 2011;94 Suppl 1: S196-S202.

27. Oike T, Ohno T, Noda SE, et al. Comparison of hematological toxicities between innovator and generic cisplatin formulations in cervical cancer patients treated with concurrent chemoradiotherapy. J Radiat Res. 2013;54(3):474-478.

28. Tsukiyama I, Hotta K, Takeuchi M, et al. Evaluation of safety in clinical use of generic paclitaxel [NK] for injection. Gan To Kagaku Ryoho. 2012;39(4):613-617.

\section{Publish your work in this journal}

Drug Design, Development and Therapy is an international, peerreviewed open-access journal that spans the spectrum of drug design and development through to clinical applications. Clinical outcomes, patient safety, and programs for the development and effective, safe, and sustained use of medicines are a feature of the journal, which

\section{Dovepress}

has also been accepted for indexing on PubMed Central. The manuscript management system is completely online and includes a very quick and fair peer-review system, which is all easy to use. Visit http://www.dovepress.com/testimonials.php to read real quotes from published authors. 